\title{
An approach to joint spectra
}

\author{
by ANGEL MARTínez MELÉNDEZ and \\ Antoni WaWrzyńczyk (México, D.F.)
}

\begin{abstract}
For a given unital Banach algebra $A$ we describe joint spectra which satisfy the one-way spectral mapping property. Each spectrum of this class is uniquely determined by a family of linear subspaces of $A$ called spectral subspaces. We introduce a topology in the space of all spectral subspaces of $A$ and utilize it to the study of the properties of the spectra.
\end{abstract}

1. Introduction. Throughout this paper we denote by $A$ a fixed unital Banach algebra with unit $e$. For every $C \subset A$ let $C^{k}$ denote the $k$ th Cartesian product of $C$. We denote by $C_{\text {com }}^{k}$ the set of all $k$-tuples of mutually commuting elements from $C$. We put $C_{\mathrm{com}}=\bigcup_{k \in \mathbb{N}} C_{\mathrm{com}}^{k}$ and $C^{\infty}=\bigcup_{k \in \mathbb{N}} C^{k}$. The norm in $A$ is denoted by $\|\cdot\|$. The algebra $A^{k}$ is provided with the norm $\left\|\left(a_{1}, \ldots, a_{k}\right)\right\|=\sup _{1 \leq i \leq k}\left\|a_{i}\right\|$. For $a=\left(a_{1}, \ldots, a_{k}\right)$ we denote by $I_{A}^{l}(a)$ (resp. $\left.I_{A}^{r}(a)\right)$ the left (resp. right) ideal generated by the elements $a_{1}, \ldots, a_{k}$ in $A$ and by $G(a)$ the unital subalgebra of $A$ generated by the same elements. We also set $P(a)=I_{G(a)}(a)$. All elements of $P(a)$ are of the form $p\left(a_{1}, \ldots, a_{k}\right)$, where $p$ is a polynomial of $k$ variables without the constant term. For $\lambda=\left(\lambda_{1}, \ldots, \lambda_{k}\right) \in \mathbb{C}^{k}$ the $k$-tuple $\left(a_{1}-\lambda_{1}, \ldots, a_{k}-\lambda_{k}\right)$ is written as $a-\lambda e$. If $a=\left(a_{1}, \ldots, a_{k}\right)$ and $b=\left(b_{1}, \ldots, b_{m}\right)$ then $(a, b)$ will denote the $(k+m)$-tuple $\left(a_{1}, \ldots, a_{k}, b_{1}, \ldots, b_{m}\right)$. For a single element $a \in A$ we denote by $\sigma(a)$ the usual spectrum of $a$.

In this paper we understand by joint spectrum a mapping $\widetilde{\sigma}$ which assigns to every $k$-tuple $a=\left(a_{1}, \ldots, a_{k}\right) \in A_{\text {com }}^{k}$ a subset $\widetilde{\sigma}(a) \subset \mathbb{C}^{k}$ not always empty such that

$$
\widetilde{\sigma}(a) \subset \prod_{i=1}^{k} \sigma\left(a_{i}\right)
$$

1991 Mathematics Subject Classification: 46H05, 46H10.

Key words and phrases: Banach algebra, joint spectrum, spectral subspace.

Research of A. Martínez partially supported by CONACyT.

Research of A. Wawrzyńczyk partially supported by SNI. 
and

$$
p(\widetilde{\sigma}(a)) \subset \widetilde{\sigma}(p(a))
$$

for every polynomial mapping $p: \mathbb{C}^{k} \rightarrow \mathbb{C}^{m}$ and every $a \in A_{\text {com }}$.

The second condition is called the one-way spectral mapping formula.

Unfortunately this terminology is not completely consistent with the axiomatics introduced by W. Zelazko in [8] in which this term is reserved for the perfect objects of spectral theory.

Recall that according to this axiomatics $\widetilde{\sigma}$ is a joint spectrum, or simply a spectrum if $\widetilde{\sigma}(a)$ is compact, satisfies (1), coincides with $\sigma(a)$ for single $a \in A$ and has the (complete) spectral mapping property:

$$
p(\widetilde{\sigma}(a))=\widetilde{\sigma}(p(a))
$$

for all $a \in A_{\text {com }}$ and all polynomial mappings $p$.

The mapping $\widetilde{\sigma}$ is called a subspectrum if (1) and (3) are satisfied.

A special case of the spectral mapping property is the projection property

$$
\pi(\widetilde{\sigma}(a))=\widetilde{\sigma}(\pi(a))
$$

where $\pi\left(x_{1}, \ldots, x_{n}\right)=\left(x_{i_{1}}, \ldots, x_{i_{m}}\right)$ for $1 \leq i_{1} \leq \ldots \leq i_{m} \leq n$.

The following translation property can be deduced from the one-way spectral mapping property:

$$
\widetilde{\sigma}(a+\lambda e)=\widetilde{\sigma}(a)+\lambda
$$

for $a \in A_{\text {com }}^{k}$ and $\lambda \in \mathbb{C}^{k}$.

A semispectrum, according to [8], is a mapping $\widetilde{\sigma}$ which satisfies (1), (4) and (5).

Finally, a generalized joint spectrum is defined on the whole $A^{\infty}$ and satisfies the conditions (1) and (2).

It was proved in [8] that every subspectrum $\tau$ on an Abelian algebra $A$ is uniquely determined by a subset $K$ of the space $M(A)$ of the multiplicative functionals on $A$ by means of the formula

$$
\begin{aligned}
\tau(a) & =\left\{\left(\varphi\left(a_{1}\right), \ldots, \varphi\left(a_{k}\right)\right) \mid \varphi \in K\right\} \\
& =\left\{\lambda \in \mathbb{C}^{k} \mid \exists \varphi \in K, a-\lambda e \in(\operatorname{ker} \varphi)^{k}\right\} .
\end{aligned}
$$

Following this idea we propose to describe in a similar form and for noncommutative $A$ a wider class of joint spectra. In place of the maximal ideals $\operatorname{ker} \varphi$ which appear in the above formula we introduce families of linear subspaces of the algebra $A$ called spectral subspaces. With every set $\mathcal{U}$ of spectral subspaces we associate a joint spectrum denoted by $\sigma_{\mathcal{U}}$.

The algebraic structure of a spectral subspace ensures that the one-way spectral mapping formula is satisfied by $\sigma_{\mathcal{U}}$. Other properties of spectra like compactness, the projection formula and the spectral mapping property depend on the structure of $\mathcal{U}$. 
We define a topology $\mathcal{T}$ in the space of spectral subspaces in such a way that $\mathcal{T}$ restricted to the set of maximal two-sided ideals coincides with the Gelfand topology. It results that for the joint spectrum $\sigma_{\mathcal{U}}$ the formula $\sigma_{\overline{\mathcal{U}}}(a)=\overline{\sigma_{\mathcal{U}}(a)}$ is valid for all $a \in A_{\text {com }}$. This formula implies that the compact spectra are exactly those defined by compact sets of spectral spaces.

Using the topology $\mathcal{T}$ we obtain in Section 4 an elementary proof of a theorem which is an extension of results of A. Soltysiak about the existence of multiplicative functionals on $A$. A generalized joint spectrum $\tau=\sigma_{\mathcal{U}}$ is nonvoid for every $a \in A^{\infty}$ if and only if $\overline{\mathcal{U}}$ contains the kernel of a multiplicative linear form on $A$.

In Section 5 we study the relation between the projection property and the spectral mapping property. The principal result states that for all joint spectra the projection property implies the spectral mapping formula without assuming the compactness of the spectrum.

In Section 6 we compare our approach to joint spectra with the method used by V. Kordula and V. Müller [3] and by V. Müller [4] based on the concepts of regularity and joint regularity.

The authors are very indebted to the reviewer for important observations which enabled them to improve the paper.

\section{Spectral linear subspaces in $A$}

Definition 1. A linear subspace $E \subset A$ is called spectral if it does not

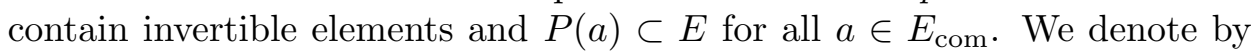
$\mathbf{E}(A)$ the set of all spectral linear subspaces in $A$.

The space $\mathbf{E}(A)$ contains in particular all left and right ideals of $A$ and all subalgebras consisting of noninvertible elements.

An example of a spectral subspace which is not a subalgebra can be constructed in the algebra of $3 \times 3$ complex matrices. Let $E$ be the subspace of all matrices of the form

$$
T(\alpha, \beta, \gamma)=\left(\begin{array}{ccc}
\alpha+\beta & \gamma & 0 \\
\gamma & \alpha-\beta & 0 \\
0 & 0 & 0
\end{array}\right) \quad \text { for } \alpha, \beta, \gamma \in \mathbb{C} .
$$

If $T, S \in E$ commute then one of them is a linear combination of the other and of the matrix $T(1,0,0)$. All powers of an element $T \in E$ belong to $E$. It follows that $E$ is a spectral subspace. On the other hand $T(0,1,0) T(0,0,1)$ $\notin E$, hence $E$ is not an algebra.

Every set $\mathcal{U}$ of spectral linear subspaces defines a joint spectrum for all $a \in A^{k}$ by the formula

$$
\sigma_{\mathcal{U}}(a)=\left\{\lambda \in \mathbb{C}^{k} \mid \exists E \in \mathcal{U}, a-\lambda e \in E^{k}\right\} .
$$


The above formula makes sense for all $a \in A^{\infty}$ but in general the most important properties of the spectrum are valid only for elements of $A_{\text {com }}$.

Proposition 2.1. For every $\mathcal{U} \subset \mathbf{E}(A)$ the spectrum $\sigma_{\mathcal{U}}(a)$ has the following properties:

(i) $\sigma_{\mathcal{U}}(a) \subset \prod_{i=1}^{k} \sigma\left(a_{i}\right)$ for $a=\left(a_{1}, \ldots, a_{k}\right) \in A^{k}$,

(ii) the translation property: $\sigma_{\mathcal{U}}(a+\lambda e)=\sigma_{\mathcal{U}}(a)+\lambda$ for $a \in A^{k}$ such that $\sigma_{\mathcal{U}}(a)$ is nonvoid and for all $\lambda \in \mathbb{C}^{k}$,

(iii) the one-way projection property: if $a \in A^{k}, \lambda \in \mathbb{C}^{k}, b \in A^{m}, \mu \in \mathbb{C}^{m}$ and $(\lambda, \mu) \in \sigma_{\mathcal{U}}(a, b)$ then $\lambda \in \sigma_{\mathcal{U}}(a)$,

(iv) the one-way spectral mapping property $p\left(\sigma_{\mathcal{U}}(a)\right) \subset \sigma_{\mathcal{U}}(p(a))$ for all $a \in A_{\mathrm{com}}^{k}$ and every polynomial mapping $p: \mathbb{C}^{k} \rightarrow \mathbb{C}^{m}$.

Proof. The properties (i)-(iii) are obvious. Let $\lambda \in \sigma_{\mathcal{U}}(a)$ for some $a \in A_{\text {com. }}^{k}$. There exists $E \in \mathcal{U}$ such that $a-\lambda e \in E^{k}$. By the remainder theorem (see [2], p. 461) we know that

$$
p(a)-p(\lambda) \in I_{G(a)}(a-\lambda e)^{m} \subset P(a-\lambda e)^{m} \subset E^{m} .
$$

It follows that $p(\lambda) \in \sigma_{\mathcal{U}}(p(a))$.

Proposition 2.1 says that $\sigma_{\mathcal{U}}$ is a joint spectrum.

All joint spectra are of this form.

Proposition 2.2. Let $\tau$ be a joint spectrum. Let $\mathcal{U}$ be the set of all linear subspaces $E \in \mathbf{E}(A)$ such that for all $a \in E_{\mathrm{com}}^{k}$ the spectrum $\tau(a)$ contains zero. Then

$$
\tau(a)=\sigma_{\mathcal{U}}(a)
$$

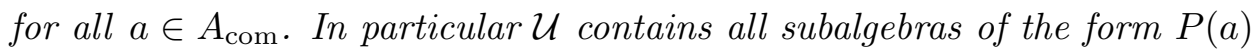
for $a \in A_{\text {com }}$ such that $0 \in \tau(a)$.

Pr o of. Both spectra $\tau$ and $\sigma_{\mathcal{U}}$ have the translation property (ii), hence in order to prove that they are identical it suffices to show that zero belongs to $\tau(a)$ if and only if it belongs to $\sigma_{\mathcal{U}}(a)$.

Take $a \in A_{\text {com }}^{k}$ such that $0 \in \tau(a)$. Let $p=\left(p_{1}, \ldots, p_{m}\right)$ be a polynomial mapping of $k$ variables without constant terms. By the one-way spectral mapping property,

$$
0=p(0) \in p(\tau(a)) \subset \tau(p(a)) .
$$

This shows that $P(a) \in \mathcal{U}$ and $0 \in \sigma_{\mathcal{U}}(a)$. The translation property yields $\tau(a) \subset \sigma_{\mathcal{U}}(a)$. The opposite inclusion follows from the definition of $\mathcal{U}$.

Proposition 2.2 and the formula (6) enable us to extend an arbitrary joint spectrum, defined initially on $A_{\text {com }}$, to the whole $A^{\infty}$ conserving at least the properties (i)-(iii) from Proposition 2.1.

The representation of joint spectra in the form $\tau=\sigma_{\mathcal{U}}$ can also be used to classify them according to the properties of the corresponding family $\mathcal{U}$. 
Repeating exactly the arguments in the proofs of Propositions 2.1 and 2.2 we obtain

Proposition 2.3. A joint spectrum $\tau$ satisfies the one-way spectral mapping property for all $a \in A^{\infty}$ if and only if there exists a subset $\mathcal{U} \subset \mathbf{E}(A)$ consisting of subalgebras of $A$ such that $\tau(a)=\sigma_{\mathcal{U}}(a)$ for all $a \in A^{\infty}$.

Proposition 2.3 describes all generalized joint spectra. In a similar way we obtain

Proposition 2.4. A joint spectrum $\tau$ can be represented as $\sigma_{\mathcal{I}}$, where $\mathcal{I}$ is a family of left (right) ideals in $A$, if and only if $\tau$ satisfies the following condition: for every $a \in A^{k}$ such that $0 \in \tau(a)$ and for every $c \in I_{A}^{l}(a)^{m}$ (resp. $c \in I_{A}^{r}(a)^{m}$ ) one has $0 \in \tau(c)$.

In Sections 4 and 5 we characterize in a similar way the joint spectra which have the complete spectral mapping property.

It is quite obvious that the same joint spectrum can be defined by different families of spectral subspaces. However, if the sets $\mathcal{U}_{\alpha}, \alpha \in I$, lead to the same spectrum and $\mathcal{U}=\bigcup_{\alpha} \mathcal{U}_{\alpha}$, then $\sigma_{\mathcal{U}}=\sigma_{\mathcal{U}_{\alpha}}$ for every $\alpha$.

This means that for a fixed joint spectrum $\tau=\sigma_{\mathcal{U}}$ there exists a unique maximal family $\mathcal{U}_{\mathrm{c}}$ such that $\tau(a)=\sigma_{\mathcal{U}_{\mathrm{c}}}(a), a \in A_{\text {com }}$. The family $\mathcal{U}_{\mathrm{c}}$ is just the union of all families which lead to the same joint spectrum $\tau$. We refer to $\mathcal{U}_{\mathrm{c}}$ as the completion of the family $\mathcal{U}$. We say that $\mathcal{U}$ is complete if it is equal to its completion.

$\mathcal{U}_{\mathrm{c}}$ can also be described in the following way:

Proposition 2.5.

$$
\begin{aligned}
\mathcal{U}_{\mathrm{c}} & =\left\{E \in \mathbf{E}(A) \mid \forall a \in E_{\mathrm{com}} \exists F \in \mathcal{U}, a \in F_{\mathrm{com}}\right\} \\
& =\left\{E \in \mathbf{E}(A) \mid 0 \in \sigma_{\mathcal{U}}(a) \forall a \in E_{\mathrm{com}}\right\} .
\end{aligned}
$$

The proof is obvious.

The maximal family corresponding to a given joint spectrum $\tau$ has appeared in the proof of Proposition 2.2. It just consists of all $E \in \mathbf{E}(A)$ such that $0 \in \tau(a)$ for every $a \in E_{\text {com }}$.

Every complete family $\mathcal{U}$ of spectral subspaces is uniquely determined by its maximal elements. If $E_{\alpha} \in \mathcal{U}$ is an increasing family of spectral subspaces then $\bigcup_{\alpha} E_{\alpha} \in \mathcal{U}$. By the Kuratowski-Zorn Lemma, $\mathcal{U}$ contains maximal elements. It follows from Proposition 2.5 that every spectral subspace $F$ which is a subset of an element $E$ of a complete family $\mathcal{U}$ is also an element of $\mathcal{U}$. Denoting by $M(\mathcal{U})$ the set of all maximal elements of a complete family $\mathcal{U}$ we obtain

$$
\mathcal{U}=\{E \in \mathbf{E}(A) \mid \exists F \in M(\mathcal{U}), E \subset F\} .
$$

The following property of joint spectra is of great importance. 
Theorem 2.1. Let $\mathcal{U} \subset \mathbf{E}(A)$. For every $a \in A^{\infty}$ define $\tau(a)=\overline{\sigma_{\mathcal{U}}(a)}$. Then there exists a family $\mathcal{V} \subset \mathbf{E}(A)$ such that $\tau(a)=\sigma_{\mathcal{V}}(a)$.

Proof. It is sufficient to prove that $\tau$ is a joint spectrum, that is, has the properties (1) and (2). The property (1) is obvious by the definition. The one-way spectral mapping formula holds for $\sigma_{\mathcal{U}}$, hence for every polynomial mapping $p$ and $a \in A_{\text {com }}$ we have

$$
p(\tau(a))=p\left(\overline{\sigma_{\mathcal{U}}(a)}\right)=\overline{p\left(\sigma_{\mathcal{U}}(a)\right)} \subset \overline{\sigma_{\mathcal{U}}(p(a))}=\tau(p(a)) .
$$

By Proposition 2.2 the assertion follows.

In the next section we define a topology in $\mathbf{E}(A)$ such that the map $\mathcal{U} \mapsto \mathcal{V}$ becomes the closure operation corresponding to this topology.

3. A topology in $\mathbf{E}(A)$. Compactness of the spectrum $\sigma_{\mathcal{U}}(a)$. Fix $E \in \mathbf{E}(A)$. For every $a \in(E+\mathbb{C} e)^{k}$ there exists a unique $\lambda_{a}(E) \in \mathbb{C}^{k}$ such that $a-\lambda_{a}(E) e \in E^{k}$. Obviously $\sigma_{\{E\}}(a)=\left\{\lambda_{a}(E)\right\}$ and $\sigma_{\mathcal{U}}(a)=\left\{\lambda_{a}(E) \mid\right.$ $E \in \mathcal{U}\}$. Let $\mathcal{T}$ be the weakest topology on $\mathbf{E}(A)$ for which the functions

$$
\lambda_{a}: E \mapsto \lambda_{a}(E) \in \mathbb{C}^{k}
$$

are continuous on their domains for all $k \in \mathbb{N}$ and all $a \in A^{k}$.

The sets $\mathcal{O}_{a, \varepsilon}(E)=\left\{F \in \mathbf{E}(A)|| \lambda_{a}(E)-\lambda_{a}(F) \mid<\varepsilon\right\}, 0 \neq a \in$ $(E+\mathbb{C} e)^{\infty}$, are a base of neighbourhoods of $E$ of the topology $\mathcal{T}$.

The topology $\mathcal{T}$ is not Hausdorff. Every element $F \in \mathbf{E}(A)$ which contains $E \in \mathbf{E}(A)$ belongs to each neighbourhood of $E$. Nevertheless, if neither of the spaces $E, F \in \mathbf{E}(A)$ is contained in the other, then they can be separated in the topology $\mathcal{T}$.

Denote by $\mathcal{U} \mapsto \overline{\mathcal{U}}$ the closure operation with respect to the topology $\mathcal{T}$.

Proposition 3.1. Let $\mathcal{U} \subset \mathbf{E}(A)$. Then

(i) $\overline{\mathcal{U}}$ is a complete family.

(ii) For every $\mathcal{U} \subset \mathbf{E}(A)$ and $a \in A_{\mathrm{com}}$,

$$
\sigma_{\overline{\mathcal{U}}}(a)=\overline{\sigma_{\mathcal{U}}(a)}
$$

Proo f. The formula $(\overline{\mathcal{U}})_{\mathrm{c}}=\overline{\mathcal{U}}$ follows from Proposition 2.5. If $\lambda \in \sigma_{\overline{\mathcal{U}}}(a)$ for $a \in A_{\text {com }}^{k}$ then there exists $E \in \overline{\mathcal{U}}$ such that $a-\lambda e \in E^{k}$. This means by the definition of $\mathcal{T}$ that for every $b \in(E+\mathbb{C} e)_{\text {com }}^{l}$ and $\varepsilon>0$ there exists $F \in \mathcal{U}$ such that $\left|\lambda_{b}(E)-\lambda_{b}(F)\right|<\varepsilon$. Since $a \in(E+\mathbb{C} e)_{\text {com }}^{k}$ and $\lambda=\lambda_{a}(E)$ this implies that $\lambda \in \overline{\sigma_{\mathcal{U}}(a)}$. The inclusion $\sigma_{\overline{\mathcal{U}}}(a) \subset \overline{\sigma_{\mathcal{U}}(a)}$ is proved.

By Theorem 2.1, $\overline{\sigma_{\mathcal{U}}(a)}=\sigma_{\mathcal{V}}(a)$ for some $\mathcal{V} \subset \mathbf{E}(A)$. The elements of $\mathcal{V}$ are defined as spectral subspaces $E \subset A$ such that for every $a \in E^{k}$ the spectrum $\sigma_{\mathcal{U}}(a)$ has zero as an accumulation point. Explicitly, this means that there exists a sequence $\mathbb{C}^{k} \ni \lambda^{(n)}(a) \rightarrow 0$ and $E_{n} \in \mathcal{U}$ such that 
$a-\lambda^{(n)}(a) e \in E_{n}$. By the definition of the topology $\mathcal{T}$ it follows that $E \in \overline{\mathcal{U}}$. We have proved in this way that $\mathcal{V} \subset \overline{\mathcal{U}}$ and hence $\overline{\sigma_{\mathcal{U}}(a)} \subset \sigma_{\overline{\mathcal{U}}}(a)$.

Corollary 3.1. If $\mathcal{U} \subset \mathbf{E}(A)$ is closed in $\mathbf{E}(A)$ then $\sigma_{\mathcal{U}}(a)$ is closed for every $a \in A_{\mathrm{com}}$. If $\sigma_{\mathcal{U}}(a)$ is closed for every $a \in A_{\mathrm{com}}$ then there exists a closed set $\mathcal{V} \subset \mathbf{E}(A)$ such that $\sigma_{\mathcal{U}}(a)=\sigma_{\mathcal{V}}(a)$.

Corollary 3.2. If $\mathcal{U}, \mathcal{V} \subset \mathbf{E}(A)$ and $\sigma_{\mathcal{U}}(a) \subset \sigma_{\mathcal{V}}(a)$ for all $a \in A_{\text {com }}$ then $\overline{\mathcal{U}} \subset \overline{\mathcal{V}}$.

Proof. As we have seen in the proof of Proposition 3.1 the closures $\overline{\mathcal{U}}$ and $\overline{\mathcal{V}}$ are the sets of elements $E$ of $\mathbf{E}(A)$ such that $\overline{\sigma_{\mathcal{U}}(a)}\left(\operatorname{resp} . \overline{\sigma_{\mathcal{V}}(a)}\right)$ contains zero for every $a \in E^{k}$.

By our assumption $\overline{\sigma_{\mathcal{U}}(a)} \subset \overline{\sigma_{\mathcal{V}}(a)}$. The assertion follows.

Fix $E \in \mathbf{E}(A)$ and $c \in E^{m}$. Let $a \in A_{\text {com }}^{k} \backslash(\mathbb{C} e)^{k}$. We introduce

$$
\mathcal{Q}_{(c, a)}=\left\{F \in \mathbf{E}(A) \mid c \in F^{m} \text {, and } \exists \lambda, a-\lambda e \in F^{k}\right\} .
$$

Proposition 3.2. The sets of the form $\mathcal{Q}_{(c, a)}$ are compact.

Proof. Let $c=\left(c_{1}, \ldots, c_{m}\right)$ and $a=\left(a_{1}, \ldots, a_{k}\right)$. Let $\left\{U_{\alpha}\right\}$ be a family of subsets of $\mathcal{Q}_{(c, a)}$ which are closed in $\mathcal{Q}_{(c, a)}$. Suppose that this family has the finite intersection property. Let $\bar{U}_{\alpha}$ denote the closure of $U_{\alpha}$ in $\mathbf{E}(A)$. Obviously the family $\left\{\bar{U}_{\alpha}\right\}$ has the finite intersection property as well.

The sets $\sigma_{U_{\alpha}}(a) \subset \mathbb{C}^{k}$ are nonempty and have the finite intersection property, hence the sets $\sigma_{\bar{U}_{\alpha}}(a)=\overline{\sigma_{U_{\alpha}}(a)}$ which are bounded and closed have a nontrivial intersection. If $\mu \in \sigma_{\bar{U}_{\alpha}}(a)$ for all $\alpha$ then $(0, \mu) \in \sigma_{\bar{U}_{\alpha}}(c, a)$ for all $\alpha$ because every element of $\mathcal{Q}_{(c, a)}$ contains all $c_{i}, 1 \leq i \leq m$. This means that the set $S=\bigcap_{\alpha} \sigma_{\bar{U}_{\alpha}}(c, a)$ is nonempty and contains an element of the form $(0, \mu)$.

For every $b \in A_{\text {com }}$ we define

$$
\tau(b)=\bigcap_{\alpha} \sigma_{\bar{U}_{\alpha}}(b) .
$$

The property $\tau(b) \subset \prod_{i} \sigma\left(b_{i}\right)$ is obvious. For every polynomial mapping $p$ we have

$$
p(\tau(b))=p\left(\bigcap_{\alpha} \sigma_{\bar{U}_{\alpha}}(b)\right) \subset \bigcap_{\alpha} p\left(\sigma_{\bar{U}_{\alpha}}(b)\right) \subset \bigcap_{\alpha} \sigma_{\bar{U}_{\alpha}}(p(b))=\tau(p(b)) .
$$

The map $\tau$ is a joint spectrum such that $\tau(c, a) \neq \emptyset$. If $(0, \mu) \in \tau(a)$ and $x=(c, a)-(0, \mu) e$ then $0 \in \tau(x)$ and $0 \in \sigma_{\bar{U}_{\alpha}}(x)$ for every $\alpha$. Since $\bar{U}_{\alpha}$ is a closed family it follows that $P(x) \in \bar{U}_{\alpha}$ by Proposition 2.2. On the other hand we observe that $P(x) \in \mathcal{Q}_{(c, a)}$. The sets $U_{\alpha}$ are closed in $\mathcal{Q}_{(c, a)}$, hence $P(x) \in U_{\alpha}$ for every $\alpha$. This proves the compactness of $\mathcal{Q}_{(c, a)}$. 
4. Joint spectra and multiplicative functionals. In this section we consider joint spectra extended to the whole space $A^{\infty}$ according to the formula (6). In general the one-way spectral mapping property is not valid in this case. Nevertheless, according to Proposition 2.1 the extended spectrum $\sigma_{\mathcal{U}}$ obeys the translation formula (4) and has the one-way projection property.

In the case of a joint spectrum defined by a closed family $\mathcal{U} \subset \mathbf{E}(A)$ we prove that $\sigma_{\mathcal{U}}(a)$ is nonvoid for all $a \in A^{\infty}$ if and only if there exists on $A$ a linear multiplicative functional $\varphi \operatorname{such}$ that $\operatorname{ker} \varphi \in \mathcal{U}$. In this way we generalize and complete the investigations initiated by C.-K. Fong and A. Soltysiak in [1] and developed by A. Soltysiak [5]-[7], concerning the existence of multiplicative functionals on $A$. Our proof is elementary, based on the theorem of Gleason-Kahane-Żelazko.

Next we prove that a generalized joint spectrum of the form $\sigma_{\mathcal{U}}$ defined on the whole $A^{\infty}$ has the spectral mapping property if and only if $M(\mathcal{U})$ consists of two-sided ideals maximal in $A$.

Both results are consequences of the following

Theorem 4.1. Let $\mathcal{U}$ be a closed subset of $\mathbf{E}(A)$. Let $E \in \mathcal{U}$. Then the following conditions are equivalent:

(i) There exists $W \in \mathcal{U}$ such that $\operatorname{codim} W=1$ and $E \subset W$.

(ii) For every $c \in E^{k}$ and every $a \in A^{m}$ there exists $\lambda \in \mathbb{C}^{m}$ such that $(0, \lambda) \in \sigma_{\mathcal{U}}(c, a)$.

Pr o o f. If (i) is valid then $A=W+\mathbb{C} e$ and there exists a linear functional $\varphi$ on $A$ such that $b-\varphi(b) e \in W$ for all $b \in A$. Given $c \in E^{k}$ and $a=$ $\left(a_{1}, \ldots, a_{m}\right)$ set $\lambda=\left(\varphi\left(a_{1}\right), \ldots, \varphi\left(a_{m}\right)\right)$; then $\lambda$ witnesses (ii).

Suppose now that (ii) is valid. When applied to $c=0$ this property implies that $\sigma_{\mathcal{U}}(a) \neq \emptyset$ for all $a \in A^{\infty}$.

For given $c \in E^{k}, a \in A^{m}$ define

$$
Z_{(c, a)}=\mathcal{Q}_{(c, a)} \cap \mathcal{U}=\left\{F \in \mathcal{U} \mid \exists \lambda \in \mathbb{C}^{m},(c, a-\lambda e) \in F^{k+m}\right\} .
$$

The condition (ii) ensures that $Z_{(c, a)} \neq \emptyset$.

The one-way projection property implies

$$
Z_{\left(\left(c_{1}, c_{2}\right),\left(a_{1}, a_{2}\right)\right)} \subset Z_{\left(c_{1}, a_{1}\right)} \cap Z_{\left(c_{2}, a_{2}\right)}
$$

for all $c_{i} \in E^{k_{i}}, a_{i} \in A^{m_{i}}$.

It follows that the family $\left\{Z_{(c, a)}\right\}_{(c, a) \in E^{\infty} \times A^{\infty}}$ has the finite intersection property.

Let us also introduce for every $(c, a) \in E^{\infty} \times A^{\infty}$ the family $\mathcal{F}_{(c, a)}$ of all functions $\varphi$ on $A$ satisfying the following conditions: 
(a) There exists $F \in Z_{(c, a)}$ such that $F \subset \operatorname{ker} \varphi$ and $b-\varphi(b) e \in F$ for every $b \in F+\mathbb{C} e$.

(b) $\varphi(b) \in \sigma_{\mathcal{U}}(b)$ for every $b \in A$.

Notice that by (a) the restriction of $\varphi$ to $F+\mathbb{C} e$ is the function $b \mapsto \lambda_{b}(F)$. In particular $\varphi(e)=1$.

The set $\mathcal{F}_{(c, a)}$ is nonempty because for every $F \in Z_{(c, a)}$ we can construct a function $\varphi \in \mathcal{F}_{(c, a)}$ putting $\varphi(b)=\lambda_{b}(F)$ for $b \in F+\mathbb{C} e$. The conditions (a) and (b) are satisfied on $F+\mathbb{C} e$ and the function can be extended to $A$ arbitrarily keeping the property (b).

This construction together with the formula (7) shows that the family of all sets $\mathcal{F}_{(c, a)}$ has the finite intersection property as well.

All sets $\mathcal{F}_{(c, a)}$ are subsets of the product $K=\prod_{a \in A} \sigma_{\mathcal{U}}(a)$, which is a compact space with respect to the product topology.

We now show that the sets $\mathcal{F}_{(c, a)}$ are closed in $K$.

Let $\varphi \in K$ be a limit of a net $\varphi_{\alpha} \in \mathcal{F}_{(c, a)}$ and let $F_{\alpha} \in Z_{(c, a)}$ witness (a) for $\varphi_{\alpha}$ on $F_{\alpha}$. By Proposition 3.2 there exists $V \in \mathcal{Q}_{(c, a)}$ and a subnet $F_{\beta}$ such that $V=\lim _{\beta} F_{\beta}$. The space $V$ is an element of $Z_{(c, a)}$, because $\mathcal{U}$ is closed. By the definition of the topology in $\mathbf{E}(A)$ for every $b \in(V+\mathbb{C} e)_{\mathrm{com}}^{m}$ and for $\beta$ "sufficiently large", $\lambda_{b}\left(F_{\beta}\right)$ is well defined and converges to $\lambda_{b}(V)$.

It follows that for $b \in V$ we have $\varphi(b)=\lim _{\beta>\beta_{0}} \varphi_{b}\left(F_{\beta}\right)=\lambda_{b}(V)=0$. The space $V$ is contained in the kernel of $\varphi$. Now, for $b \in V+\mathbb{C} e$,

$$
\varphi(b)=\lim _{\beta>\beta_{0}} \varphi_{\beta}(b)=\lim _{\beta>\beta_{0}} \lambda_{b}\left(F_{\beta}\right)=\lambda_{b}(V) .
$$

This implies that $b-\varphi(b) e \in V$ and the conditions (a) and (b) are satisfied for $\varphi$. We have proved that the set $\mathcal{F}_{(c, a)}$ is closed.

By the compactness of $K$ there exists $\varphi \in \bigcap_{(c, a)} \mathcal{F}_{(c, a)}$. It follows that the whole space $E$ is contained in $\operatorname{ker} \varphi$. For every $b \in A^{k}$ there exists $F \in Z_{(0, b)} \subset \operatorname{ker} \varphi$ such that $\varphi(x)=\lambda_{x}(F)$ and $x-\varphi(x) e \in F$ for $x \in F+\mathbb{C} e$. The function $x \mapsto \lambda_{x}(F)$ is linear on $F+\mathbb{C} e$, hence in particular on the space $\langle b\rangle$ linearly generated by the elements $b_{1}, \ldots, b_{k}$. This proves that $\varphi$ is linear everywhere.

If $b \in(\operatorname{ker} \varphi)_{\text {com }}^{k}$ then by the above property $b \in F^{k}$ for some $F \in Z_{(0, b)}$. It follows that $W=\operatorname{ker} \varphi \in \mathcal{U}$, because $\mathcal{U}$ is complete.

Corollary 4.1. Let $\mathcal{U} \subset \mathbf{E}(A)$ be a closed subset. Then $\sigma_{\mathcal{U}}(a) \neq \emptyset$ for all $a \in A^{\infty}$ if and only if there exists a linear multiplicative functional $\mu$ on $A$ such that $\operatorname{ker} \mu \in \mathcal{U}$.

P r o of. It suffices to apply Theorem 4.1 in the case of $E=0$. There exists a linear form on $A$ whose kernel is an element of $\mathcal{U}$. By the Gleason-KahaneŻelazko theorem every linear form which vanishes uniquely on non-invertible elements is multiplicative. 
CoROllary 4.2. Let $\tau$ be a joint spectrum defined on $A^{\infty}$ such that $\tau(a)$ is compact for all $a \in A^{\infty}$. Let $\mathcal{U} \subset \mathbf{E}(A)$ be the maximal family such that $\tau(a)=\sigma_{\mathcal{U}}(a)$. The spectral mapping property is valid for $\tau$ if and only if $M(\mathcal{U})$ consists of maximal two-sided ideals. This being the case,

$$
\tau\left(a_{1}, \ldots, a_{k}\right)=\left\{\left(\varphi_{I}\left(a_{1}\right), \ldots, \varphi_{I}\left(a_{k}\right)\right) \mid I \in M(\mathcal{U})\right\} .
$$

We have denoted by $\varphi_{I}$ the multiplicative functional corresponding to the maximal ideal $I$.

Pr o of. Assume that $\tau$ has the spectral mapping property. The condition (ii) in Theorem 4.1 is a special case of the formula (3), hence every element $I$ maximal in $\mathcal{U}$ is of codimension 1 in $A$. Let $\varphi$ be the linear form such that $\varphi(e)=1$ and $\operatorname{ker} \varphi=I$. By the theorem of Gleason-KahaneŻelazko $\varphi$ is multiplicative and hence $I$ is a maximal two-sided ideal in $A$. Now, if $a-\lambda e \in E^{k} \subset I^{k} \in M(\mathcal{U})^{k}$ then by the definition of $\varphi_{I}$ we obtain $\lambda_{i}=\varphi_{I}\left(a_{i}\right)$. The formula (8) is proved.

If we suppose that $M(\mathcal{U})$ consists of ideals which are maximal in $A$ then (8) is valid and the spectrum defined by the right hand side of (8) has the spectral mapping property.

5. The spectral mapping property. In this section we study the case of a joint spectrum $\tau$ which has the complete spectral mapping property for all $a \in A_{\text {com }}^{k}$, which means that for every polynomial mapping $p: \mathbb{C}^{k} \rightarrow \mathbb{C}^{m}$ we have

$$
p(\tau(a))=\tau(p(a))
$$

A special case of the spectral mapping property is the projection property (4), which in the case of a joint spectrum is equivalent to the following condition: for every $a \in A_{\text {com }}^{k}, \lambda \in \tau(a)$ and $b \in A^{m}$ such that $(a, b) \in A_{\text {com }}$ there exists $\mu \in \mathbb{C}^{m}$ such that $(\lambda, \mu) \in \tau(a, b)$.

If we represent $\tau$ as $\sigma_{\mathcal{U}}$ where $\mathcal{U} \subset \mathbf{E}(A)$ is complete then the projection property of $\tau$ is equivalent to the following property of $\mathcal{U}$ : for every $E \in \mathcal{U}$, $a \in E_{\mathrm{com}}^{k}$ and $b \in A^{m}$ such that $(a, b) \in A_{\mathrm{com}}^{k+m}$ there exist $E_{1} \in \mathcal{U}$ and $\mu \in \mathbb{C}^{m}$ such that $(a, b-\mu e) \in E_{1}^{k+m}$.

This property of $\mathcal{U}$ will also be called the projection property.

Lemma 5.1. Let $\mathcal{U}, \mathcal{V} \subset \mathbf{E}(A)$ with $\mathcal{U} \subset \mathcal{V}$. Suppose that $\sigma_{\mathcal{U}}$ has the projection property and $\sigma_{\mathcal{V}}$ has the spectral mapping property. Then $\sigma_{\mathcal{U}}$ also has the spectral mapping property.

Pr o o f. The one-way spectral mapping property is valid for $\sigma_{\mathcal{U}}$, hence it remains to prove that $\sigma_{\mathcal{U}}(p(a)) \subset p\left(\sigma_{\mathcal{U}}(a)\right)$ all $a \in A_{\text {com }}^{k}$ and all polynomial mappings $p$. Suppose that $\lambda \in \sigma_{\mathcal{U}}(p(a))$. By the projection property of $\mathcal{U}$ there exists $\mu \in \mathbb{C}^{k}$ such that $(\lambda, \mu) \in \sigma_{\mathcal{U}}(p(a), a)$. The one-way spectral 
mapping property implies $\mu \in \sigma_{\mathcal{U}}(a)$. The inclusion $\mathcal{U} \subset \mathcal{V}$ and the spectral mapping formula for $\sigma_{\mathcal{V}}$ yield

$$
(\lambda, \mu) \in \sigma_{\mathcal{U}}(p(a), a) \subset \sigma_{\mathcal{V}}(p(a), a)=\left\{(p(\mu), \mu) \mid \mu \in \sigma_{\mathcal{V}}(a)\right\} .
$$

It follows that all elements of the spectrum $\sigma_{\mathcal{U}}(p(a), a)$ have the form $(p(\mu), \mu)$ for $\mu \in \sigma_{\mathcal{U}}(a)$. We obtain $\lambda=p(\mu)$, which was to be proved.

Proposition 5.1. Assume that the joint spectrum $\tau$ has the projection property. Then $\bar{\tau}(a)=\overline{\tau(a)}$ is also a joint spectrum with the projection property.

Pro of. It was proved in Theorem 2.1 that $\bar{\tau}$ is a joint spectrum. Let $\lambda \in$ $\bar{\tau}(a)$ for some $a \in A_{\text {com }}^{k}$ and let $(a, b) \in A_{\text {com }}^{k+m}$. There exists a sequence $\lambda(n) \in$ $\tau(a)$ which converges to $\lambda$. By the projection property applied to $\tau$ there exists $\mu(n) \in \mathbb{C}^{m}$ such that $(\lambda(n), \mu(n)) \in \tau(a, b)$. The sequence $(\lambda(n), \mu(n))$ has a convergent subsequence whose limit $(\lambda, \mu)$ belongs to $\bar{\tau}(a, b)$. The assertion follows.

COROLlary 5.1. If a joint spectrum $\tau$ on a Banach algebra $A$ has the projection property, then it has the spectral mapping property.

P r o of. Let $a \in A_{\mathrm{com}}^{k}$. Let $B$ be the maximal unital Abelian subalgebra of $A$ which contains $a_{1}, \ldots, a_{k}$. The joint spectrum $\tau$ restricted to $B$ is a joint spectrum on $B$ with the projection property. Denote it by $\tau_{1}$. By Proposition 5.1, $\bar{\tau}_{1}$ has the projection property, and by Corollary 4.2, it has the spectral mapping property. Lemma 5.1 implies that $\tau_{1}$ itself has the spectral mapping property, which ensures the same property for $\tau$.

6. Regularities and joint spectra. The papers [3] by V. Kordula and V. Müller and [4] by V. Müller describe wide classes of spectra and joint spectra using the concepts of regularities and of joint regularities.

According to [3] a regularity in $A$ is a nonempty subset $R$ of $A$ which satisfies the following conditions:

(i) if $a \in A$ and $n \in \mathbb{N}$ then $a \in R$ if and only if $a^{n} \in R$,

(ii) if $(a, b, c, d) \in A_{\text {com }}^{4}$ and $a c+b d=e$, then $a b \in R$ if and only if $a \in R$ and $b \in R$.

A regularity defines a spectrum for single elements $a \in A$ by the formula

$$
\widetilde{\sigma}_{R}(a)=\{\lambda \in \mathbb{C} \mid a-\lambda e \notin R\} .
$$

In general $\widetilde{\sigma}_{R}(a)$ is neither compact nor non-empty. One of the most important properties of the spectrum $\widetilde{\sigma}$ proved in [3] is the following

Theorem 6.1. Let $R$ be a regularity in $A$. Then for all $a \in A$ and for every function $f$ analytic in a neighbourhood of $\sigma(a)$ and nonconstant on 
every connected component of its domain,

$$
\widetilde{\sigma}_{R}(f(a))=f\left(\widetilde{\sigma}_{R}(a)\right) .
$$

Note. If $0 \notin R$ then the one-way spectral mapping formula is also valid for the constant function $f$.

Not every spectrum of the form $\widetilde{\sigma}_{R}$ can be extended to a subspectrum. A necessary condition is that $a b \in R$ should imply $a \in R$ and $b \in R$ for all $a, b \in A$. It is not satisfied in general because there exist examples of regularities which contain zero. If we exclude this case we can always extend $\widetilde{\sigma}_{R}$ to a semispectrum.

Proposition 6.1. Let $R$ be a regularity in $A$ such that $0 \notin R$. Let $\mathcal{J}$ be the set of all subalgebras of $A$ of the form $P(a), a \in R^{\mathrm{c}}$. Then

$$
\sigma_{\mathcal{J}}(a)=\widetilde{\sigma}_{R}(a)
$$

for $a \in A$ and

$$
p\left(\sigma_{\mathcal{J}}(a)\right) \subset \sigma_{\mathcal{J}}(p(a))
$$

for every $a \in A^{\infty}$ and every polynomial mapping $p$.

Proof. If $0 \in \widetilde{\sigma}_{R}(a)$ for $a \in A$ then $a \in P(a) \in \mathcal{J}$, which means that $0 \in \sigma_{\mathcal{J}}(a)$.

If $0 \in \sigma_{\mathcal{J}}(a)$, then there exists a polynomial $p$ without constant term and an element $b \notin R$ such that $a=p(b)$. By the spectral mapping formula, $\widetilde{\sigma}_{R}(a)=p\left(\widetilde{\sigma}_{R}(b)\right)$. Since $0 \in \widetilde{\sigma}_{R}(b)$ we obtain $0 \in \widetilde{\sigma}_{R}(a)$. Both spectra have the translation property, hence they coincide.

The joint spectrum $\sigma_{\mathcal{J}}$ is defined by means of a family of subalgebras of $A$, hence by Proposition 2.3 it has the one-way spectral mapping property for all $k$-tuples in $A$.

In [4], V. Müller has introduced the concept of joint regularity and of spectral system, which is just the joint spectrum associated with a joint regularity.

Definition 2. A joint regularity is a subset $R \subset A_{\text {com }}$ of the form $R=\bigcup_{n=1}^{\infty} R_{n}$, where $R_{n} \subset A_{\text {com }}^{n}$ and for all $n \in \mathbb{N}$ the following conditions are satisfied: $\in R_{n}$.

(i) If $\left(x_{1}, \ldots, x_{n}, y_{1}, \ldots, y_{n}\right) \in A_{\text {com }}$ and $\sum_{i=1}^{n} x_{i} y_{i}=e$ then $\left(x_{1}, \ldots, x_{n}\right)$

(ii) If $\left(x_{1}, \ldots, x_{n}, x_{n+1}\right) \in A_{\text {com }}$ and $\left(x_{1}, \ldots, x_{n}\right) \in R_{n}$ then $\left(x_{1}, \ldots\right.$ $\left.\ldots, x_{n}, x_{n+1}\right) \in R_{n+1}$.

(iii) If $\left(x_{0}-\lambda, x_{1}, \ldots, x_{n}\right) \in R_{n+1}$ for every $\lambda \in \mathbb{C}$ then $\left(x_{1}, \ldots, x_{n}\right) \in R_{n}$. 
The joint spectrum associated with the regularity $R$ is defined for $x \in A_{\text {com }}^{n}$ as

$$
\widetilde{\sigma}_{R}(x)=\left\{\lambda \in \mathbb{C}^{n} \mid x-\lambda e \notin R_{n}\right\} .
$$

The map $\widetilde{\sigma}_{R}$ is an example of a spectral system.

Definition 3. A spectral system for $A$ is a mapping which assigns to every $x \in A_{\text {com }}^{k}$ a subset $\widetilde{\sigma}(x)$ which has the projection property (4) and satisfies the condition $\tilde{\sigma}(x) \subset \sigma^{G(x)}(x)$, where the latter set is the usual joint spectrum of $x$ in the Abelian algebra $G(x)$.

As proved in [4] there exists a one-to-one correspondence between joint regularities and spectral systems. Formula (10) describes the spectral system associated with a given regularity $R$. Conversely, if for a given spectral system $\widetilde{\sigma}$ we define $R_{n}=\left\{x \in A_{\text {com }}^{n} \mid 0 \notin \widetilde{\sigma}(x)\right\}$ then $\widetilde{\sigma}(x)=\widetilde{\sigma}_{R}$ ([4], Theorem 5). All spectral systems have the spectral mapping property.

TheOREM 6.2 ([4], Theorem 7). Let $\widetilde{\sigma}$ be a spectral system in the algebra A. Let $x \in A_{\text {com }}$ and let $f=\left(f_{1}, \ldots, f_{k}\right)$ be a $k$-tuple of functions analytic in some neighbourhood of $\sigma^{G(x)}(x)$. Then

$$
\widetilde{\sigma}(f(x))=f(\widetilde{\sigma}(x)) .
$$

The definitions of regularity and of joint regularity admit the case of $R=$ $A_{\text {com }}$, which corresponds to $\widetilde{\sigma}_{R}(x)=\emptyset$ for all $x$. Excluding this (pathological, in our opinion) case we can describe the regularities and spectral systems in terms of spectral subspaces in $A$.

TheOREM 6.3. Let $\widetilde{\sigma}=\widetilde{\sigma}_{R}$ be a spectral system such that $R \neq A_{\mathrm{com}}$. Let

$$
\mathcal{U}=\left\{E \in \mathbf{E}(A) \mid \forall k \in \mathbb{N}, x \in E_{\text {com }}^{k}, 0 \in \widetilde{\sigma}(x)\right\} .
$$

Then the family $\mathcal{U}$ has the projection property, $\sigma_{\mathcal{U}}(x)=\widetilde{\sigma}(x)$ and

$$
R_{n}=A_{\mathrm{com}}^{n} \backslash\left(\bigcup_{E \in \mathcal{U}} E^{n}\right) .
$$

Proof. Let $x \in A$ and $0 \in \tilde{\sigma}(x)$. Assume that $x$ is invertible. By the projection property of $\widetilde{\sigma}$ there exists $\mu \in \mathbb{C}$ such that $(0, \mu) \in \widetilde{\sigma}\left(x, x^{-1}\right) \subset$ $\sigma^{G\left(x, x^{-1}\right)}\left(x, x^{-1}\right)$. This is a contradiction which shows that $x$ is noninvertible and $P(x) \in \mathcal{U}$.

The equality $\widetilde{\sigma}=\sigma_{\mathcal{U}}$ follows from Proposition 2.2. The projection property of $\mathcal{U}$ is now obvious since $\widetilde{\sigma}$ has the spectral mapping property. Comparing the definition of the regularity associated with $\widetilde{\sigma}$ with the definition of $\sigma_{\mathcal{U}}$ we obtain the description (11) of $R_{n}$ in terms of elements of spectral subspaces belonging to $\mathcal{U}$.

If we describe spectral systems in terms of regularities we must construct an infinite family of sets $R_{n}$ satisfying 3 axioms. Using for this purpose 
spectral subspaces we must determine only a set of subspaces $E \subset R_{1}$ such that $R_{1}=\bigcap_{E \in \mathcal{U}} E^{\mathrm{c}}$ and then the higher regularities are uniquely described by formula (11).

\section{References}

[1] C.-K. Fong and A. Sołtysiak, Existence of a multiplicative functional and joint spectra, Studia Math. 81 (1985), 213-220.

[2] R. Harte, Invertibility and Singularity for Bounded Linear Operators, Marcel Dekker, New York, 1988

[3] V. Kordula and V. Müller, On the axiomatic theory of spectrum, Studia Math. 119 (1996), 109-128.

[4] V. Müller, Spectral systems, unpublished notes, 1997.

[5] A. Soltysiak, Approximate point joint spectrum and multiplicative functionals, Studia Math. 86 (1987), 227-286

[6] - Joint spectra and multiplicative functionals, Colloq. Math. 56 (1989), 357-366.

[7] _, Joint Spectra and Multiplicative Linear Functionals in Non-commutative Banach Algebras, Wyd. Nauk. Uniw. im. A. Mickiewicza, Poznań, 1988.

[8] W. Żelazko, An axiomatic approach to joint spectra I, Studia Math. 64 (1979), 249-261.

Angel Martínez Meléndez

Antoni Wawrzyńczyk

Universidad Autónoma

Universidad Autónoma

Metropolitana - Azcapotzalco

Metropolitana - Iztapalapa

Departamento de Ciencias Básicas

Av. San Pablo 180

col. Reynosa Tamaulipas

02200 México, D.F., México

E-mail: amm@hp9000a1.uam.mx

Av. Michoacán y la Purísima s/n col. Vicentina AP 55-538

09340 México, D.F., México

E-mail: awaw@xanum.uam.mx 